\title{
FAMILIES OF RATIONAL SURFACES PRESERVING A CUSP SINGULARITY
}

\author{
LEE J. MCEWAN
}

\begin{abstract}
Families of rational surfaces containing resolutions of cusp singularities are explicitly constructed. It is proved that the families constructed are universal deformations at each point. Two different monodromy formulas are established; one of these is shown to be connected to automorphisms of InoueHirzebruch surfaces. Some evidence (but no proof) is offered for the conjecture that finite base changes of the families we construct are the versal-deformation spaces for singular Inoue-Hirzebruch surfaces.
\end{abstract}

\section{INTRODUCTION}

Let $(Y, D)$ consist of the following data: $Y$ is a rational surface, $D \in|-K|$ a reduced divisor consisting of smooth $P^{1}$ 's meeting normally in a cycle such that the components of $D$ generate a negative definite lattice $(K$ a canonical divisor). $\quad D$ is usually called an anticanonical cycle. We refer to [9] for the importance and general facts of such surfaces. In this paper we construct explicitly and investigate topologically general smooth families of pairs $(Y, D)$, where $D$ is fixed in a lattice sense.

Here is an outline of the paper: $\S 1$ gives a review of relevant facts from [9]. In $\S 2$ we apply a theorem of Miranda (Theorem 2.1) which allows us to realize any pair $(Y, D)$ as a blowup of $\left(P^{2}, F\right), F$ being 3 lines in general position, in a systematic way. The sequence of blowups factors into two types:

(1) Blowing up at a node ("corner blowup"), and

(2) blowing up at a smooth point ("interior blowup").

The sequence can be arranged so that all type (2)'s occur after all type (1)'s. Knowing this sequence determines the lattice-embedding $\left\langle D_{i}\right\rangle \rightarrow H_{2}(Y ; Z)$ of the span of the components of $D$ in $H_{2}$ of the surface.

Let $(\bar{Y}, \bar{D})$ be the intermediate surface obtained after performing all the corner blowups. We construct a base-space for a family from a product of $\mathbf{C}^{*}$ 's, corresponding to the components of $\bar{D}$ on which we make interior blowups. The space $M$ that we construct simply parametrizes the continuous choices we must make in the sequence connecting $Y$ to $\bar{Y}$. Next we form the product

Received by the editors January 20, 1987 and, in revised form, November 8, 1988. Preliminary version presented at the Charlotte, N.C., meeting of the A.M.S., October 1986.

1980 Mathematics Subject Classification (1985 Revision). Primary 14B05, 14B07, 14D05, 14J26, 14J17, 32G05, 32G13; Secondary 14J05, 14J15, 14J50, 57R50, 57R52.

(C) 1990 American Mathematical Society $0002-9947 / 90 \$ 1.00+\$ .25$ per page 
$\bar{Y} \times M$; regarding each point of $M$ as a locus of blowups in $\bar{Y}$, we obtain sections of $\bar{Y} \times M \rightarrow M$. Blowing up on these sections gives a family $\mathbf{Y}$ over $M$.

In $\S 3$ we specialize to the case where $M$ is one-dimensional; all our subsequent constructions generalize easily, but in fact we expect the one-dimensional case to have the most applications. In this case $M$ is isomorphic to $\mathbf{C}^{*}$. Using a handle-body decomposition (our basic tool) we compute the monodromy on the open surface $Y \backslash D$ (Theorem 3.9); the answer is in terms of the data of $D$ and $(\bar{Y}, \bar{D})$. Next we prove (Theorem 3.10) that the family is a universal deformation space in the sense of Looijenga; we use the handle-body decomposition to compute the period map explicitly. Applying the decomposition again, we obtain a monodromy formula on $H_{1}(\tau ; Z)$ (Theorem 3.13), where $\tau$ is a 3-manifold which appears on the boundary of a regular neighborhood of $D$ in $Y$. The action on $\tau$ itself is interesting: We describe it as a "generalized Dehn-twist" and the resulting monodromy formula as a "Picard-Lefschetz transformation." Next we show (using the results of [11]) that this formula is the same as that induced by the action (3.12) of the (finite) group of complex automorphisms of a certain nonalgebraic surface $X$ (we construct $X$ in $\S 1$ ). $X$ is the so-called Inoue-Hirzebruch surface which exists for any (abstractly given) $D$; it contains $D$ and another cycle called the dual of $D$.

From this observation we draw some positive evidence for a conjecture stated in [9]. Let us state the conjecture and describe our evidence.

Given any (abstract) cycle $D$-i.e., a cycle of $P^{1}$ 's with assigned self-intersections and generating a negative lattice, but independent of any embedding in a rational surface-we may still construct the surface $X$. Let $X^{\prime}$ be the surface obtained from $X$ by contracting the cycle dual to $D$ to a singular point. Looijenga has shown that the existence of any smoothing of just the germ of the singularity implies that $X^{\prime}$ deforms in a family of rational surfaces, each with an anticanonical cycle $D$, and such that some neighborhood of $D$ is preserved analytically on every fiber.

He conjectures the converse: Suppose $D$ embeds in a rational surface as an anticanonical cycle. Then $X^{\prime}$ lives in a family as described above (and thus the contracted dual to $D$ has a smoothing).

We have shown that $\operatorname{Aut}(X)$ acts on neighborhoods of $D$ in our family; to get a family which satisfies some of Looijenga's conditions we base-change our family to order $|\operatorname{Aut}(X)|$. This eliminates the monodromy around $D$ (Corollary 3.14) and gives a family which is topologically trivial near $D$-and one on which $\operatorname{Aut}(X)$ acts in the base. This fits with the conjecture since $\operatorname{Aut}(X)$ also acts on $X^{\prime}$, and therefore on any family in which $X^{\prime}$ deforms. Finally, a theorem of Laufer (Theorem 1.2) implies that our family is locally analytically trivial near $D$. Our results thus give some positive evidence for the truth of the conjecture, as well as suggest that our (base-changed) family is the family $X^{\prime}$ smooths in. 
$\S 4$ concludes with some numerical relations between the various quantities in the construction, as well as some consequences for the intersection matrix of an anticanonical cycle which sits on a rational surface in a one-dimensional family.

I express here my deep thanks to my teachers at Columbia University, most especially to Henry Pinkham, without whose endless and varied support I could not have produced this work.

\section{1}

We start with some preliminary definitions and known results which provide the motivation for what we've done.

(1.1) Definition. A cusp singularity is a normal surface singularity with a minimal resolution consisting of either a nodal rational curve or a cycle of smooth $P^{1}$ s.

Or course, the lattice spanned by the components of the resolution must be negative definite. This condition is in turn equivalent to assuming that each component $D_{i}$ of the resolution satisfies $D_{i}^{2}<-1$, and at least one $D_{i}^{2}<-2$, or that $D^{2}<0$ in the case of one component.

\section{(1.2) Theorem (Laufer [7]). Cusp singularities are taut.}

This fact is essential for our study of rational surfaces degenerating to the Inoue.

There is a notion of duality among cusps. We will use the notation of [4] to express the algorithm discovered by Hirzebruch-Zagier. (Then rather than describe the duality intrinsically we will use the output of the algorithm as the definition of the dual.)

A cusp with minimal resolution $D=\bigcup_{i=1}^{n} D_{i}$, where $D_{i} D_{j}=1$ iff $|i-j|=1$ (taking $i$ and $j \bmod (n))$, can be described by a $2 \times k$ matrix

$$
\left|\begin{array}{l}
a_{i} \\
b_{i}
\end{array}\right|
$$

where the $a_{i}$ are the negatives of the squares of those components which satisfy $D_{i}^{2}<-2$ (and $k$ equals the number of such components), and $b_{i}$ equals the number of components (each having square equal -2 ) between two successive components with square less than -2 . Then the dual $C$ to $D$ is given by

$$
C=\left|\begin{array}{l}
b_{i}+3 \\
a_{i}-3
\end{array}\right| \text {. }
$$

We will construct only a portion of the Inoue surface $X$, namely a neighborhood of the resolution $D$. The reader needs to know only that $X$ consists of two such neighborhoods (the other a neighborhood of the dual $C$ ), glued along the complements of $C$ and $D$ themselves. 
Before proceeding to the construction we list some of the important properties of $X$.

(1) The algebraic dimension of $X$ is zero.

(2) $X$ possesses the (disjoint) minimal resolutions of two (dual) cusps as its only curves. Their union is anticanonical.

(3) $b_{1}$ (the first Betti number of $X$ ) is 1 .

It follows from (3) that $X$ is non-Kahler.

Now for the construction. Start with a cusp $D=\cup D_{i}$, as above; it is determined by an ordered sequence of positive integers $\left[d_{1}, \ldots, d_{n}\right]$, where $d_{i}=-D_{i}^{2}$.

Take an infinite series of copies of $\mathbf{C}^{2}$, indexed by $\mathbf{Z}$ :

$$
V_{i}=\left\{\left(u_{i}, v_{i}\right) \in \mathbf{C}^{2}\right\}, \quad i \in \mathbf{Z} .
$$

Glue the $V_{i}$ together by the rule

$$
v_{i}=v_{i-1}^{d_{i-1}} \cdot u_{i-1}, \quad u_{i}=1 / v_{i-1},
$$

where $d_{i}=d_{[i]},[i]=$ residue of $i(\bmod n)$.

Let $V$ be the union of the glued $V_{i}$. By Hirzebruch [6, Proposition 2.1] $V$ is Hausdorff and therefore a complex manifold.

Notice that on the open sets $\left\{u_{i} v_{i}=0\right\}$, the equations (1.3) are invertible; the space $V$ is thought of as a partial compactification of $\left\{u_{0} v_{0}=0\right\} \approx \mathbf{C}^{*} \times \mathbf{C}^{*}$, with an infinite chain of $P^{1}$ 's added on at the boundary. Let $\widetilde{D}=\bigcup_{i=-\infty}^{\infty} D_{i}=$ $\bigcup_{i=-\infty}^{\infty}\left\{v_{i}=0\right\}$ denote this chain.

Define an automorphism of $V$ by

$$
g: V_{i} \rightarrow V_{i-n}, \quad\left(u_{i}, v_{i}\right) \rightarrow\left(u_{i}, v_{i}\right)
$$

(1.4) Proposition (Inoue [6]). There exists a connected neighborhood $F$ of $\widetilde{D}$ which is preserved by $g$, and on which it acts properly discontinuously without fixed points.

We divide $F$ by the infinite-cyclic group generated by $g$ : the result is an open complex manifold which contains $D:=\widetilde{D} / g$, a (resolved) cusp singularity.

There is a number-theoretic point of view which we will also need. More details of the following, including proofs, can be found in [5] and [6]. Let $w$ be defined by the following modified continued fraction:

$$
w=d_{1}-\frac{1}{d_{2}-\frac{1}{d_{3}} \cdots} .
$$

Then $w$ is in fact a quadratic irrational number: $w=a+b \sqrt{d}, a$ and $b$ rational and $d \in \mathbf{Z}, d$ positive and square-free. $w$ generates an extension of the rational numbers $\mathbf{Q}$ which is of degree two. Denote the involution (i.e., the 
nontrivial generator of the Galois group of the extension) by a prime $\left(^{\prime}\right)$. Then in fact $w>1>w^{\prime}>0$. Define the following objects:

$$
\begin{aligned}
M & =M(w)=\mathbf{Z} \text {-module generated by }\{1, w\} ; \\
U & =U(m)=\text { group of positive units of } M \\
& =\{\beta \mid \beta M(w)=M(w) \text { and } \beta>0\} ; \\
U^{+} & =U^{+}(M)=\text { totally positive units of } M \\
& =\left\{\alpha \in U \mid \alpha>0 \text { and } \alpha^{\prime}>0\right\} .
\end{aligned}
$$

Then $U$ and $U^{+}$are infinite-cyclic groups under multiplication, and $\left[U: U^{+}\right.$] $=1$ or 2 .

Let $G$ be the semidirect product of $M$ and $U^{+}, G$ acts on $\mathbf{H} \times \mathbf{H}$ (where $\mathbf{H}$ is the upper half plane) as follows:

$$
\begin{aligned}
& \left(z_{1}, z_{2}\right) \rightarrow\left(z_{1}+m, z_{2}+m^{\prime}\right), \quad m \in M, \\
& \left(z_{1}, z_{2}\right) \rightarrow\left(\alpha z_{1}, \alpha^{\prime} z_{2}\right), \quad \alpha \in U^{+} .
\end{aligned}
$$

Let $z_{1}=x_{1}+i y_{1}, z_{2}=x_{2}+i y_{2} ; \mathbf{H} \times \mathbf{H}$ fibers over $\mathbf{R}_{+}$(the positive real numbers) with fiber

$$
W_{d}=\left\{\left(z_{1}, z_{2}\right) \mid y_{1} \cdot y_{2}=d\right\} .
$$

Then $G$ acts on each $W_{d}$; in fact, $W_{d}$ itself fibers over the real line $y_{1} y_{2}=d$, and $G$ acts equivariantly on this fibration:

$$
W_{d} / G \rightarrow\left\{\left(y_{1}, y_{2}\right) \mid y_{1} y_{2}=d\right\} / U^{+} \approx S^{1} .
$$

The fiber of the quotient-fibration is a real 2-torus. The three-manifold $W_{d} / G$ is a nontrivial torus bundle which will be called $\tau_{d}$; its monodromy can be computed directly from the cycle $\left[d_{1}, \ldots, d_{n}\right]$. The quotient of $\mathbf{H} \times \mathbf{H}$ by $G$ can be written $\mathbf{H} \times \mathbf{H} / G=\bigcup_{d \in \mathbf{R}} \tau_{d}$. We partially compactify the quotient by adding on curves at the "end" $d=+\infty$. Recall that $V \backslash \widetilde{D}$ is just $\mathbf{C}^{*} \times \mathbf{C}^{*}$ and that $\left(u_{0}, v_{0}\right)$ are coordinates for it. The equations

$$
\begin{aligned}
& 2 \pi i z_{1}=w \log \left(v_{0}\right)+\log \left(u_{0}\right), \\
& 2 \pi i z_{2}=w^{\prime} \log \left(v_{0}\right)+\log \left(u_{0}\right)
\end{aligned}
$$

identify $\mathbf{H} \times \mathbf{H}$ with a portion of $V \backslash \widetilde{D}$, which is just the space $F \backslash \widetilde{D}$. Taking quotients on both sides, (1.5) induces an isomorphism:

$$
\Phi:(F \backslash \widetilde{D}) / g \rightarrow \mathbf{H} \times \mathbf{H} / G .
$$

By patching this space to the quotient of $\mathbf{H} \times \mathbf{H}$ via $\Phi$ we have added the cycle $D$ to the latter.

Remark. By making $G$ act on $\mathbf{H} \times \mathbf{C}$ instead of $\mathbf{H} \times \mathbf{H}$ we obtain the entire Inoue surface except for the cycles $C$ and $D$. A similar patching construction is needed to add the cycle $C$, and the final result is compact.

We will almost always be concerned with the surface obtained from $X$ by contracting one or both of these cycles, to obtain a singular surface. 


\section{(1.6) Definition.}

$$
\begin{aligned}
& X^{\prime}=X \text { after contracting } C \text { to a point } c . \\
& X^{\prime \prime}=X \text { after contracting } C \text { and } D \text { to points } c \text { and } d .
\end{aligned}
$$

(1.7) Proposition (Looijenga [9, III, (2.3)]). $X^{\prime \prime}$ admits a universal deformation $q: \mathbf{X} \rightarrow T$ which restricts to a semiuniversal deformation of the (disconnected) germ of the two singularities $\{c, d\}$.

This proposition is a formal consequence of the computation $H^{i}\left(\theta_{X^{\prime \prime}}\right)=0$ for all $i$.

From this proposition it follows that any smoothing of $c$ can be realized by a deformation of $X^{\prime \prime}$, which we may further assume is rigid "away from $c$ ": Let $S$ be the subvariety of $T$ whose fibers preserve the singularity $d$. Any smoothing of $c$ must be represented in $S$. The singularity $d$ on each fiber defines a section of the bundle $\left.\mathbf{X}\right|_{S}$; minimal resolution along this section gives us a family $\mathbf{Y} \stackrel{p}{\rightarrow} S$, with a divisor $\mathbf{D}$ which restricts to a cycle $D_{t}:=\mathbf{D} \cdot Y_{t}$ of rational curves on each fiber $Y_{t}=p^{-1}(t), t \in S$. By the following proposition (and its preceding discussion) $(\mathbf{Y}, \mathbf{D})$ is a universal deformation (preserving $D)$ for $\left(X^{\prime}, D\right)$, and $D_{t}$ is anticanonical on $Y_{t}$ :

(1.8) Proposition [9, III, (2.8)]. Let $S_{f}$ denote the set of $t \in S$ such that $Y_{t}$ has only rational double points as singularities. Then for any $t \in S_{f}, Y_{t}$ is a rational surface equipped with a negative definite anticanonical cycle $D_{t}$ and $\mathbf{Y}$ is a universal deformation of the pair $\left(Y_{t}, D_{t}\right)$.

Therefore, the smoothability of $c$ implies the existence of a smooth rational surface on which the (resolved) dual of $c$ sits as an anticanonical cycle. On the other hand, the existence of such a surface is always decidable, given the data of the dual (which can be computed from that of $c$ by the Hirzebruch-Zagier algorithm).

(1.9) Theorem (Wahl [12]). Let $r$ be the number of components in the minimal resolution of $c$. Then the dimension of any smoothing component of $\left(X^{\prime}, c\right)$ is $10+C \cdot C+r$.

Finally, we have Looijenga's conjecture:

(1.10) Assume $10+C \cdot C+r>0$. Then $\left(X^{\prime}, c\right)$ is smoothable if there exists a smooth rational surface with anticanonical cycle $D$ (with $D$ dual to $C$ ).

This conjecture has been proved for $r \leq 3$ by Friedman-Pinkham [4] and in other cases by Looijenga [9]. Our program is to construct the only candidate for the smoothing and to compute the basic monodromy information associated to it. Then we can show that the family (after finite base change) is topologically trivial in a neighborhood of $D$. By tautness of the singularity $D$, we have local analytic triviality. The crucial question is how large a neighborhood of $D$ 
can be kept as we go to the boundary. This deeper question remains unsolved by us.

\section{MOdUli SPACE OF RATIONAL PAIRS}

In this section we construct the base space for a family of rational surfaces with a fixed anticanonical cycle. These surfaces are polarized in the sense of coming equipped with a specified blowdown to $P^{2}-$ i.e., "the way $D$ sits on $Y$ " is part of the construction. The base space of the family is a universal deformation space for such surfaces. Let $\left[d_{1}, \ldots, d_{n}\right]$ be a fixed sequence of positive integers, with $d_{i} \geq 2$ and $d_{i}>2$ for at least one $i$. We will also assume initially that $n \geq 4$, but our eventual assumption of a 1-dimensional modulus forces $n$ to be much larger. We begin with a basic theorem due to Rick Miranda:

(2.1) Theorem (Rick Miranda). Suppose that $Y$ is a smooth rational surface with an anticanonical cycle $D$ with intersection sequence $\left[d_{1}, \ldots, d_{n}\right], n \geq 4$. Then $Y$ can be blown down to $P^{1} \times P^{1}$ so that $D$ is mapped to the standard square, $F=\left(P^{1} \times\{0, \infty\}\right) \cup\left(\{0, \infty\} \times P^{1}\right)$.

(2.2) Corollary. With the same assumption as in Theorem 2.1, Y can be blown down to $P^{2}$, sending $D$ to three lines in general position.

Proof. Blow down to $P^{1} \times P^{1}$. To go back, we must make either an interior blowup or a corner blowup. If the former, we create a new -1-curve on the component opposite to the one we blew up on. Blow it down to obtain $F_{1}$. Then blow down again to get $P^{2}$. If we made a corner blowup, contract the new -1-component to get $F_{1}$ again. Now blow down to get $P^{2}$.

Proof of Theorem 2.1. Choose a ruling on $Y$. By Riemann-Roch a fiber in this ruling meets $D$ twice (since $D$ is anticanonical). Claim: It is possible to choose the ruling so that two disjoint components are sections in it. If this does not happen, there exists among the components of $D$ a bisection or two sections that meet. If there is a bisection, the rest of $D$ lies in a fiber. See that you can blow down to a Hirzebruch surface $F_{m}$ and get a cycle with two components, one of them a fiber. This implies $m=0,1$, or 2. (For $m>2$ the canonical system of $F_{m}$ has a fixed component with negative square.) Check that no matter how you blow up, an appropriate ruling appears by the time the cycle has four components. A similar analysis holds for two sections that meet.

Given the ruling, any singular fiber not containing components of $D$ must consist of a chain of $P^{1}$ 's with self-intersection equal to -1 for the ends, which meet $D$, and -2 for components in the middle. The fibers which contain components of $D$ can be blown down to a chain contained in $D$; then it has either a -1-curve not meeting either section, or both components on the ends are -1 's. If the former, blow down to shrink the chain. If the latter, blow down on the most negative section or until one section has square 0 , and then 
blow down on the other section until the fiber is simple. Do this for both chains containing components of $D$. Similarly, blow down each singular fiber in the ruling, always on the most negative section, or until one section has square 0 , and then always on the other section until the fiber is simple. At the end $D$ consists of four components: two fibers and two sections, $s_{1}$ and $s_{2}$. Now $s_{i}^{2}$ cannot both be negative since they are disjoint and the surface is relatively minimal, so we may assume that $s_{1}^{2}$ equals zero. Now $s_{2}^{2}<0$ is impossible since $D^{2}=8$ or $9=8+s_{2}^{2}$. Therefore $s_{2}^{2}=0$ or 1 ; but $s_{2}$ is disjoint from $s_{1}$, which defines a ruling; so $s_{2}$ is in a fiber and must satisfy $s_{2}^{2} \leq 0$. Therefore $s_{2}^{2}=0$.

Using Theorem 2.1 in reverse, we can obtain $(Y, D)$ as a sequence of blowups of $\left(P^{1} \times P^{1}, F\right)$. Factor $\pi:(Y, D) \rightarrow\left(P^{1} \times P^{1}, F\right)$ into a sequence of blowups $\pi_{j}:\left(Y_{j}, D^{j}\right) \rightarrow\left(Y_{j-1}, D^{j-1}\right)$. Each blowup falls into one of two categories:

(a) $\pi_{j}$ blows up $D^{j-1}$ at the intersection of two components (corner blowup). Then the exceptional divisor becomes a component of $D^{j}$, increasing its length by one.

(b) $\pi_{j}$ blows up $D^{j-1}$ at a smooth point (interior blowup). The exceptional divisor is transverse to $D^{j}$ and lowers $\left(D^{j}\right)^{2}$ by 1 . Of course, $\pi_{j}$ cannot blow up a point off $D$ because the exceptional locus would belong to $-K$; i.e., $D$ would no longer be anticanonical.

It is clear that we may always assume that all corner blowups are performed first, before making any interior blowups. The surface $(\bar{Y}, \bar{D})$ obtained after all the corner blowups are made is called a toroidal surface associated to $Y$ (or to the cycle $\left.\left[d_{1}, \ldots, d_{n}\right]\right)$. It is a complete torus embedding, i.e., a compactification of $\mathbf{C}^{*} \times \mathbf{C}^{*}$ to which the self-action of $\mathbf{C}^{*} \times \mathbf{C}^{*}$ extends naturally. The material at infinity is the cycle $\bar{D}$ of rational curves. Moduli for the surfaces $(Y, D)$ which dominate $(\bar{Y}, \bar{D})$ accrue by making interior blowups on $\bar{D}$. A blowup point on a component of $\bar{D}$ may vary on that component without changing the data of $D$, but in general two surfaces obtained by blowing up distinct points on the same component will not be isomorphic. (By isomorphism we mean an analytic isomorphism of pairs $(Y, D) \rightarrow\left(Y^{\prime}, D^{\prime}\right)$.) However, we must compensate for the action of $\mathbf{C}^{*} \times \mathbf{C}^{*}$ on $\bar{Y}$, which moves points on $\bar{D}$. Suppose $\bar{D}_{i}, i \in I$, are the components of $\bar{D}$ which receive interior blowups, and $m_{i}$ is the number of blowups on $\bar{D}_{i}$. Let $\bar{D}_{i}^{*}=\bar{D}_{i} \backslash\{0, \infty\}$. Then $\mathbf{C}^{*} \times \mathbf{C}^{*}$ acts on each $\bar{D}_{i}^{*} \subset \bar{Y}$ and therefore on the product $\prod_{i \in I}\left(\bar{D}_{i}^{*}\right)^{m i}$. There is a natural map

$$
\mu: \mathbf{C}^{*} \times \mathbf{C}^{*} \rightarrow \prod_{i \in I}\left(\bar{D}_{i}^{*}\right)^{m i}
$$

which sends $(z, w)$ to the image of 1 (the identity) under the corresponding action in each component. (Later we will introduce natural coordinates on $\bar{D}_{i}$.) 
The quotient by the image of $\mu$ is again a product of $\mathbf{C}^{*}$ 's. Therefore we have constructed $\mathbf{M} \approx\left(\mathbf{C}^{*}\right)^{p}$, where $p=\left(\sum_{i \in I} m_{i}\right)-2$, as a candidate for the moduli space of pairs.

Henceforth we will concentrate on the special case $\operatorname{dim} \mathbf{M}=1$.

Therefore assume $\left(\sum m_{i}\right)-2=1$, which implies exactly three interior blowups on $(\bar{Y}, \bar{D})$. $\mathbf{M}$ is described by a single parameter $t \in \mathbf{C}^{*} \approx \mathbf{M}$. For use in the next section we prove

(2.3) Lemma. Suppose $\operatorname{dim} \mathbf{M}=1$. Then $(Y, D)$ dominates a toroidal surface $(\bar{Y}, \bar{D})$ by contracting three exceptional curves of the first kind which meet distinct components of $D$.

Proof. By Corollary (2.2) we may assume that $(\bar{Y}, \bar{D})$ is obtained by blowing up $P^{2}$ at the vertices of three lines in general position. Since $\operatorname{dim} \mathbf{M}$ is 1 , there are exactly three interior blowups. Now suppose that every vertex of the original three lines in $P^{2}$ is blown up to reach $\bar{Y}$; then the last blowup at each corner creates a -1 component. These must be the curves which receive interior blowups, and they are distinct. Therefore suppose that exactly two vertices are blownup. Two distinct -1 components are then created; it is easy to check that it is impossible to push the squares of the strict transforms of the original three lines below -1 without creating a third -1 component (one must blow up on both sides of one of the first two -1 components). Finally, blowing up only one vertex is impossible: the line opposite that vertex remains with square +1 , so it requires three internal blowups. Together with the -1 component from the last corner blowup, there are too many moduli.

Let us compute the action of $\mathbf{C}^{*} \times \mathbf{C}^{*}$ on the components of $\bar{D}$ in terms of a natural set of coordinate systems, in anticipation of constructing a family of pairs $\left(Y_{t}, D_{t}\right)$ over $\mathbf{M}$. First note that we can construct $(\bar{Y}, \bar{D})$ in a manner analogous to the construction of the Inoue surface, but using only finitely many charts:

Let $\bar{d}_{i}=-\bar{D}_{i}^{2}$ on $\bar{Y}_{i}$.

Let $\left(z_{i}, w_{i}\right)$ be coordinates on $U_{i}=\mathbf{C}^{2}, i=1, \ldots, n$, where $n$ is the number of components of $D$, and make the following identifications:

$$
w_{i+1}=1 / z_{i}, \quad z_{i+1}=w_{i} z_{i}^{\bar{d}_{i}}, \quad i=1, \ldots, n,
$$

where we take $i \bmod (n)$, e.g., $U_{n+1}=U_{1},\left(z_{n+1}, w_{n+1}\right)=\left(z_{1}, w_{1}\right)$. Blowing up only at corners preserves the following relationship:

$$
\prod_{i=1}^{n}\left|\begin{array}{cc}
\bar{d}_{i} & 1 \\
-1 & 0
\end{array}\right|=\left|\begin{array}{ll}
1 & 0 \\
0 & 1
\end{array}\right|
$$

This in turn implies that equations (2.4) induce no identifications of different points in the same chart; i.e., each $U_{i}$ injects into the identification space $\bar{Y}$ via the inclusion map.

Now $\mathbf{C}^{*} \times \mathbf{C}^{*}$ acts on a (fixed) chart (say $U_{1}$ ) by

$$
(s, t):\left(z_{1}, w_{1}\right) \rightarrow\left(s z_{1}, t w_{1}\right)
$$


and on the rest of $\bar{Y}$ by transforming (2.5) into other charts via (2.4). Explicitly, the following induced action is easily checked. Let

$$
\left|\begin{array}{ll}
a_{i} & b_{i} \\
c_{i} & d_{i}
\end{array}\right|:= \begin{cases}\prod_{j=1}^{i-1}\left|\begin{array}{cc}
\bar{d}_{j-1} & 1 \\
-1 & 0
\end{array}\right| & (i>1), \\
\left|\begin{array}{cc}
1 & 0 \\
0 & 1
\end{array}\right| & (i=1) .\end{cases}
$$

Then

$$
\left(z_{i}, w_{i}\right) \rightarrow\left(s^{a_{i}} t^{b_{i}} z_{i}, s^{c_{i}} t^{d_{i}} w_{i}\right) .
$$

Now suppose that $\bar{D}_{i_{1}}, \bar{D}_{i_{2}}$, and $\bar{D}_{i_{3}}$ are the three components that receive interior blowups. Relabel in this context so that $\bar{D}_{i_{1}}=: \bar{D}_{1}, a_{i_{1}}=: a_{1}, z_{i_{1}}=: z_{1}$, etc. for these three components.

We may take $z_{i}$ as affine coordinate on $\bar{D}_{i}, i=1,2,3$. Then $\left(z_{i}\right) \in$ $\mathbf{C}^{*} \times \mathbf{C}^{*} \times \mathbf{C}^{*}$ are parameters for $\mathbf{M}$, which are acted on by $\mathbf{C}^{*} \times \mathbf{C}^{*}$ explicitly:

$$
\left(z_{i}\right) \rightarrow\left(s^{a_{i}} t^{b_{i}} z_{i}\right) \text {. }
$$

Let $p_{i} \in \mathbf{Z}, i=1,2,3$, be a primitive solution to the system

$$
\sum_{i=1}^{3} p_{i} a_{i}=0, \quad \sum_{i=1}^{3} p_{i} b_{i}=0 .
$$

Then $\left(z_{i}\right) \rightarrow(1,1,1)$ via the action if and only if $\prod_{i=1}^{3} z_{i}^{p_{i}}=1$. That is,

$$
\mathbf{C}_{z_{1}}^{*} \times \mathbf{C}_{z_{2}}^{*} \times \mathbf{C}_{z_{3}}^{*} \rightarrow \mathbf{M} \quad \text { by }\left(z_{i}\right) \rightarrow \prod_{i=1}^{3} z_{i}^{p_{i}}
$$

(2.8) Remark. We will see below (Lemma 3.7) that the Z-vectors $\left(a_{i}\right)$ and $\left(b_{i}\right)$ must be linearly independent, essentially on account of the negative-definiteness of the span of the components of $\mathrm{D}$ in $\mathrm{H}_{2}(Y ; \mathbf{Z})$. So $\left(p_{i}\right)$ is unique up to \pm 1 .

The next section concerns the construction of a family of pairs $\left(Y_{t}, D\right)$ over $\mathbf{M}$ and describes the topological structure of it.

\section{CONSTRUCTION OF THE BASIC FAMILY}

As above, let $(\bar{Y}, \bar{D})$ be a toroidal surface associated to the cycle $\left[d_{1}, \ldots\right.$, $\left.d_{n}\right]$. Recall that $\mathbf{M}$ is the cokernel of $\mu: \mathbf{C}^{*} \times \mathbf{C}^{*} \rightarrow \prod_{i=1}^{3} D_{i}^{*}$; the latter object has been identified with a product of $\mathbf{C}^{*}$ 's via the coordinate systems on $Y$. Let $p: \mathbf{M} \rightarrow \prod_{i=1}^{3} \bar{D}_{i}^{*}=\mathbf{C}_{z_{1}}^{*} \times \mathbf{C}_{z_{2}}^{*} \times \mathbf{C}_{z_{3}}^{*}$ be a section of the quotient map $\sigma: \prod_{i=1}^{3} \bar{D}_{i}^{*} \rightarrow \mathbf{M}$. Explicitly, we may let $p(t)=\left(t^{e_{1}}, t^{e_{2}}, t^{e_{3}}\right)$, where $\left(e_{1}, e_{2}, e_{3}\right) \cdot\left(p_{1}, p_{2}, p_{3}\right)=1$ and the $e_{i}$ are integers (recall that $\left.\operatorname{gcd}\left(p_{i}\right)=1\right)$. Then $\sigma\left(\left(z_{i}\right)\right)=\prod_{i=1}^{3} z_{i}^{p_{i}}$ implies that $\sigma(p(t))=t$. Let $\pi_{i}: \prod_{i=1}^{3} \bar{D}_{i}^{*} \rightarrow \bar{D}_{i}^{*}$ be the projection maps. Form the (trivial) family $\bar{Y} \times \mathbf{M} \rightarrow \mathbf{M}$ (where we project onto the second factor) and define sections $S_{i}$ of it by $S_{i}=\left(f_{i} \pi_{i} p\right.$, id. $)$, where 
$f_{i}$ is the inclusion $\bar{D}_{i}^{*} \rightarrow \bar{Y}$. By Lemma (2.3) these sections have disjoint images in the family. Blow up these sections to obtain the "basic" family $\mathbf{Y} \rightarrow \mathbf{M}$, each fiber of which maps to the point it defines in $M$.

Next we analyze this family topologically; derived from this we will establish certain monodromy formulas.

There are monodromy actions (on two different groups) that are of interest, namely on $H_{2}\left(Y_{t} \backslash D ; \mathbf{Z}\right)$ and on $H_{1}(\tau ; \mathbf{Z})$, where $\tau$ is the (3-dimensional) boundary of a regular neighborhood $N(D)$ of $D$ in $Y_{t} . \tau$ is the same space occurring in the description of the Inoue surface, i.e., a torus-bundle over a circle. (Because $D$ is taut, $N(D)$ must be isomorphic to a regular neighborhood of $D$ in the Inoue surface; in particular the boundaries of these spaces must be homeomorphic.)

If the family of rational pairs filled in with the Inoue surface $X^{\prime}$, then $Y_{t} \backslash D$ would be the Milnor fiber of the singularity, and the family of neighborhoods $N(D) \quad\left(D \subset Y_{t}\right)$ could be analytically trivialized over the base (in accordance with Looijenga). Due to the nontrivial monodromy action occurring on the boundary of $N(D)$, such a trivialization cannot exist for this family; what we show in fact is that this monodromy is always of finite order. More precisely, the order of the monodromy is equal to the order of the (finite) group of complex automorphisms of the Inoue surface. After a base change of degree equal to this common number, we obtain a family which is topologically trivial "at infinity," i.e., such that the family of neighborhoods $N(D)$ is just a product $N(D) \times \mathbf{C}^{*}$. Note that Laufer's theorem of the tautness of $D$ implies that the neighborhoods $N(D)$ are all analytically isomorphic; therefore the family of neighborhoods is locally analytically trivial. What we cannot do yet is rule out the possibility of complex analytic monodromy on the family of neighborhoods of $D$. However, according to [8, Proposition 3], the group of complex automorphisms of $N(D)$ is a Lie group with at most countably many components; presumably an automorphism which is isotopic to the identity (through a $C^{\infty}$ isotopy) is in the identity component of this group. Analytic triviality of the family would follow from this.

(3.1) Handle-body decomposition. We will describe the topological structure of $Y_{t}$ by first decomposing $\bar{Y}$ and then adding handles to the pieces, corresponding to the blowups.

Let $\bar{B}$ be a regular neighborhood of the cycle $\bar{D}$ in $\bar{Y}$, and let $\bar{A}$ be the closure of the complement of $\bar{B}$. Topologically, $\bar{A}$ is a deformation retract of $\mathbf{C}^{*} \times \mathbf{C}^{*}: \bar{A} \approx S^{1} \times S^{1} \times B^{2}$ where $B^{2}$ is the 2-disk and $S^{1}$ the 1-sphere. Think of $\bar{A}$ as a "thickened" 2-torus $\left(T^{2} \times B^{2}\right)$, and obtain its handle decomposition by thickening the usual handle-body decomposition for the 2-torus: $T^{2}=(0$-handle) $\cup$ (two 1-handles) $\cup$ (one 2-handle) (see Figure 1). Multiply each handle by $B^{2}$ to get handles of the same degree in dimension 4 . Then $\bar{A}$ has a decomposition:

$$
\bar{A}=h_{0} \cup\left\{h_{1}^{1}, h_{1}^{2}\right\} \cup h_{2}, \quad \text { where } h_{1}^{*} \text { is a 1-handle. }
$$




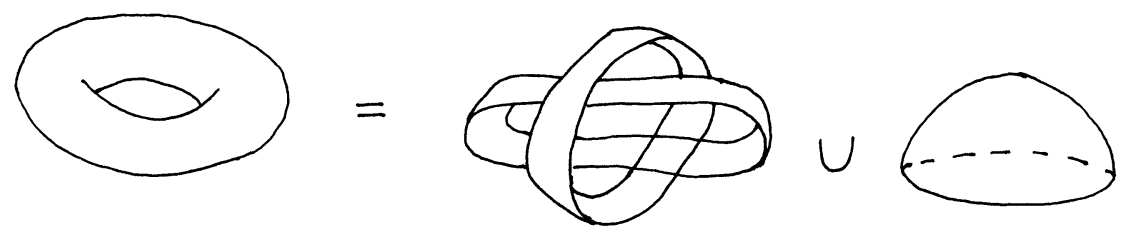

FIGURE 1

Notice that the boundary of $\bar{A}$, where we will attach more handles to get $Y_{t}$, can be thought of as a trivial $T^{2}$-fiber bundle over $S^{1}: \partial \bar{A} \approx \partial\left(T^{2} \times B^{2}\right)=$ $T^{2} \times \partial B^{2}=T^{2} \times S^{1}$. We could compute the homology of $\bar{A}$ immediately from the handle decomposition. The handles give generators in each degree; let $C_{k}$ be the free-abelian group generated by the $k$-handles. The boundary operator $\partial_{k}: C_{k} \rightarrow C_{k-1}$ is computed by forming the matrix $\left(\alpha_{i j}^{k}\right)$ where $\alpha_{i j}^{k}$ equals the algebraic intersection of the core of the $j$ th $k$-handle with the cocore of the $i$ th $(k-1)$-handle. $H_{3}$ and $H_{4}$ are zero since there are no generators in those degrees; the single 2-handle has algebraic intersection zero with each 1-handle, so $\partial_{2}=\left(\alpha_{i j}^{2}\right)=0$. (The 2-handle kills some homotopy classes but no homology classes.) $\partial_{1}$ is also zero, so there is one generator in $H_{2}(\bar{A} ; \mathbf{Z})$ and two in $H_{1}(\bar{A} ; \mathbf{Z})$. The generator of $H_{2}(\bar{A} ; \mathbf{Z})$ is the class of the fiber of the above-mentioned $T^{2}$-bundle.

In order to construct $Y_{t}$ we digress to a description of blowing up and its effect on the regular neighborhood of a curve.

(3.2) Topological description of a blowup; effect on neighborhood of a curve. Let $N$ be a neighborhood of a (smooth) point $p$ on a curve $C$, and let $R$ be a regular collar-neighborhood of the curve (see Figures 2-4).

To blow up at $p$, remove $N$ and glue $C P^{2} \backslash B^{4}$ to $\partial N$. ( $B^{4}$ is the 4-ball. $)$
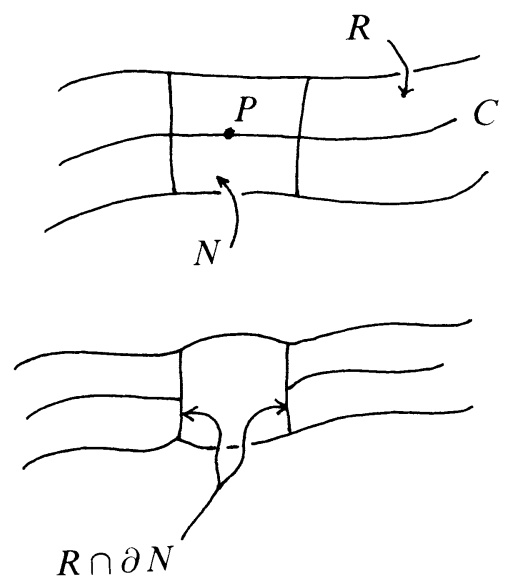

FIGURE 2 

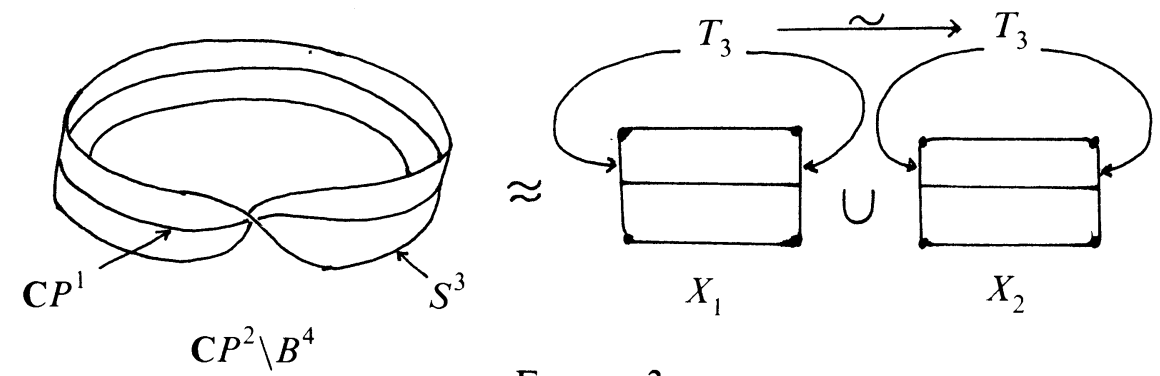

FIGURE 3

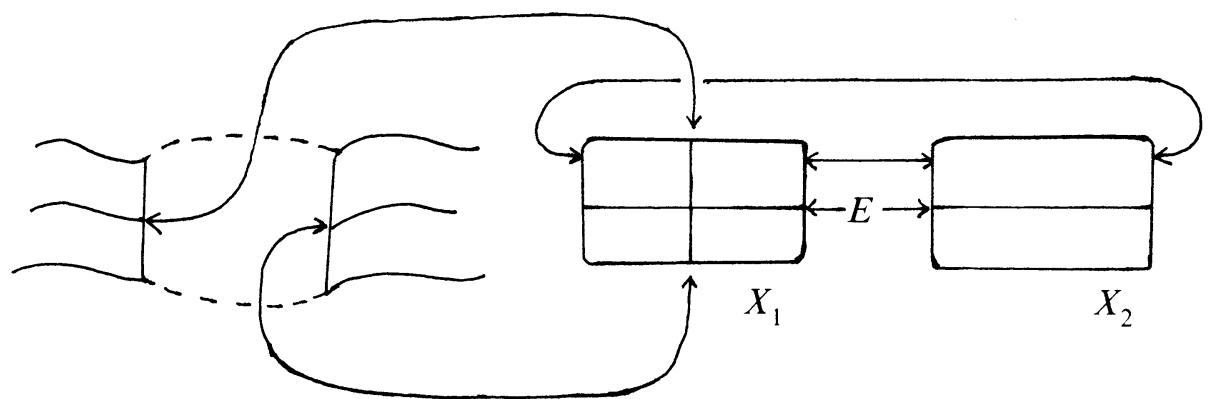

FIGURE 4

To relate this to $R$ and $\widetilde{C}$ (the strict transform of $C$ ), glue in $\mathbf{C} P^{2} \backslash B^{4}$ in two pieces: one piece to form $\widetilde{C}$ and its neighborhood, the other piece to the complement of $R$.

Decompose $\mathbf{C} P^{2} \backslash B^{4}$ into $X_{1} \cup X_{2}, X_{i} \approx B^{4}$, where the $X_{i}$ are glued along a portion of their boundaries: $\partial X_{i}=S^{3}$ (3-sphere); $S^{3}$ decomposes as two "solid tori": $S^{3} \approx\left(S^{1} \times B^{2}\right) \cup\left(B^{2} \times S^{1}\right)$. (One standard way to view this decomposition is as follows: Consider the set of lines through a point in $\mathbf{C} P^{2}$ parametrized by a line not passing through the point. We remove a neighborhood $\left(\approx B^{4}\right)$ of the point; what's left becomes a fibration of 2-disks over the line. Then decompose the line $\left(\approx S^{2}\right)$ into two 2-disks $E_{1}$ and $E_{2}$ glued along $S^{1}$ and restrict the fibration to each disk. Each restricted fibration is trivial, giving us two $B^{4}$ 's.)

The $X_{i}$ are glued along a $T^{3} \approx S^{1} \times B^{2}$ in each of their boundaries; the leftover boundary pieces are glued along their boundaries (the four vertices of the $X_{i}$ in the picture (Figure 4)) to form the $S^{3}=\partial\left(\mathbf{C} P^{2} \backslash B^{4}\right)$.

Reverse the order of the identifications by first gluing the $X_{i}$ onto $\partial N$. Arrange $R$ so that $R \cap \partial N \approx T^{3}$. Near $p, R$ fibers over $C$ with fiber $B^{2}$, and $R \cap \partial(N \cap C)=S^{1}$. Then $\partial N$ consists of two pieces, one contained in $R \backslash N$, the other not meeting $R$.

One of the $X_{i}$, say $X_{1}$, is glued to $R \cap \partial N . X_{2}$ is glued to the other portion of $\partial N$.

The exceptional curve is the cores of $X_{1}$ and $X_{2}$, or just the line in the description of $\mathbf{C} P^{2} \backslash B^{4}$ above. A fiber over $E_{1}$ in $X_{1}=B^{2} \times E_{1}$ becomes the strict transform of $C$. 
If we take the boundaries of the fiber and of the base in $X_{1}$ as an ordered basis for $H_{1}\left(\partial T^{3} ; \mathbf{Z}\right)$, and similarly for $R \cap \partial N$, then $X_{1}$ is glued to $R \backslash N$ by $\left|\begin{array}{cc}1 & -1 \\ 0 & 1\end{array}\right|$.

Now to build $Y_{t}$. Let $g_{i}$ be the points to be blown up, and choose neighborhoods $N_{i}$ of the $g_{i}$ as in the picture (see Figure 2). Decompose the boundary of each $N_{i}$ into two pieces, $\partial N_{i} \cap \bar{A}$ and $\partial N_{i} \cap \bar{B}$. Remove the $N_{i}$. As above, attach 2-handles to $\bar{A}$ and $\bar{B} \backslash\left(\bigcup N_{i}\right)$ along the $N_{i}$ boundary pieces. The space $B:=\left(\bar{B} \backslash\left(\bigcup N_{i}\right)\right) \cup$ (three 2-handles) is a regular neighborhood of $D$ in $Y_{t}$, and $A:=\bar{A} \cup\left(\cup h_{2}^{i}\right)$ is topologically equivalent to $Y_{t} \backslash D$. Now we compute $H_{2}\left(Y_{t} \backslash D ; \mathbf{Z}\right)=H_{2}(A ; \mathbf{Z})$. As before, let $C_{k}$ be the free-abelian group generated by the $k$-handles of $A$. Again, $C_{4}=C_{3}=0$, so we are reduced to computing the homology of the sequence

$$
0 \rightarrow C_{2} \stackrel{\partial_{2}}{\rightarrow} C_{1} \rightarrow 0 \text {. }
$$

Therefore

$$
H_{2}(A, \mathbf{Z})=\operatorname{ker} \partial_{2}, \quad H_{1}(A, \mathbf{Z})=C_{1} / \operatorname{im} \partial_{2}
$$

We have

$$
C_{2}=\mathbf{Z}\left\langle h_{2}^{0}, h_{2}^{1}, h_{2}^{2}, h_{2}^{3}\right\rangle, \quad C_{1}=\mathbf{Z}\left\langle h_{1}^{1}, h_{1}^{2}\right\rangle,
$$

where $h_{2}^{0}$ is the 2-handle in the decomposition of $\bar{A}$. To compute $\partial_{2}$ we relate the decomposition of $A$ to the coordinates of $\bar{Y}$. Regard $\bar{A}$ as a trivial torus bundle $\bar{\tau}=T^{2} \times S^{1}$ over $S^{1}$; a section of this bundle can be obtained by lifting $\Gamma$, a generating loop of $H_{1}(\bar{D}, \mathbf{Z}) \approx \mathbf{Z}$, into $N(\bar{D})$. (There are many ways to lift.) Recall that $\bar{Y}$ can be described as the union of $n$ copies of $\mathbf{C}^{2}$ glued together by equations of the form

$$
x_{i+1}=1 / y_{i}, \quad y_{i+1}=x_{i} y_{i}^{\bar{d}_{i}} .
$$

The equation $y_{i+1}=0$ defines the $i$ th component of $\bar{D}$, and $-\bar{d}_{i}$ is its self-intersection. We will use these equations to describe the attaching spheres of the new 2-handles. These are $S^{1}$ 's in the boundary of $\bar{A}$; in fact each one can be taken to lie on a fiber of $\bar{\tau}$. Let $p$ be one of the blowup points, $(x, y)=$ standard local coordinates, and assume that $\Gamma$ passes through $p$. Then the fiber associated to $p \in \Gamma$ may be represented by $\{(x, y)|| x|=| p|| y \mid,=$ $1\}$. We choose a marking on this fiber as

$$
\begin{aligned}
& b_{1}:=\left\{(x, y)|x=| p \mid \cdot e^{i \theta}, y=1,0 \leq \theta \leq 2 \pi\right\}, \\
& b_{2}:=\left\{(x, y) \mid x=p, y=e^{i \phi}, 0 \leq \phi \leq 2 \pi\right\} .
\end{aligned}
$$

The triple $\left\{b_{1}, b_{2}, \Gamma\right\}$ gives an ordered basis for $H_{1}(\bar{\tau}, \mathbf{Z})$. With this convention, the attaching sphere for $h_{2}$ is just $b_{2}$. The construction can be repeated at each point $p_{i} ; h_{2}^{i}$ is therefore attached to $b_{2}^{i}$ where $\left\{b_{1}^{i}, b_{2}^{i}\right\}$ is the local standard marking of the fiber associated to $p_{i}$. Now we must relate each basis to 
the handle-body decomposition of $\bar{A}$. We may assume that the two 1-handles $h_{1}^{1}$ and $h_{1}^{2}$ have cores homologous to $b_{1}^{1}$ and $b_{1}^{2}$ (respectively). We also assume that the components of $\bar{D}$ are labeled so that $p_{1}$ lies on $\bar{D}_{1}$. Recall that $\partial_{2}=\left(\alpha_{j i}\right)$, where $\alpha_{j i}=$ (core $i$ th 2-handle) $\cap$ (cocore $j$ th 1-handle). Then since $b_{2}^{1}$ is also the attaching sphere for $h_{2}^{1}$, we have $\left(\alpha_{11}, \alpha_{21}\right)=(0,1) .\left(b_{2}^{1}\right.$ passes once through the cocore of $h_{1}^{2}$ and avoids $h_{1}^{1}$.) For the other 2-handles, we need only relate the bases $\left\{b_{1}^{i}, b_{2}^{i}\right\}$ in which their attaching spheres are written to the basis defining the decomposition. (We omit the third basis element $\Gamma$, which is the same for all bases.) The change-of-basis matrix is just a product of the matrices which define the gluings: $\left|\begin{array}{cc}\bar{d}_{i} & 1 \\ -1 & 0\end{array}\right|$.

(3.5) Lemma. $x_{11} b_{1}^{1}+x_{21} b_{2}^{1}=x_{1 i} b_{1}^{i}+x_{2 i} b_{2}^{1}$ in $H_{1}(\bar{\tau}, \mathbf{Z}) \approx \mathbf{Z}^{3}$ exactly if

$$
\left(x_{1 i}, x_{2 i}\right)=\prod_{j=1}^{i-1}\left|\begin{array}{cc}
\bar{d}_{i-j} & 1 \\
-1 & 0
\end{array}\right| \cdot\left(\begin{array}{l}
x_{11} \\
x_{21}
\end{array}\right) .
$$

Proof. Apply the transformation (3.3) to the representation (3.4) corresponding to the basis $\left\{b_{1}^{i}, b_{2}^{i}\right\}$. The exponents $(\theta, \phi)$ transform by the matrices $\left|\begin{array}{cc}\bar{d}_{j} & 1 \\ -1 & 0\end{array}\right|$, $j$ ranging over the component indices intervening between $\bar{D}_{i}$ and $\bar{D}_{1}$.

Let $\left(\beta_{1 i}, \beta_{2 i}\right)$ be the coordinates of $b_{2}^{i}$ written in the basis of $\left\{b_{1}^{1}, b_{2}^{1}\right\}$. Then clearly $\alpha_{j i}=\beta_{j i}, i=1,2,3$. Note also that the attaching sphere of $h_{2}^{0}$ is just $b_{1} b_{2} b_{1}^{-1} b_{2}^{-1}$, i.e., "zero" homologically. Therefore $\alpha_{j 0}=0, j=1,2$.

Now we can compute $\partial_{2}$. We see by Lemma 3.5 that

$$
\left(x_{1 i}, x_{2 i}\right)=(0,1)=\prod_{j=1}^{i-1}\left|\begin{array}{cc}
\bar{d}_{i-j} & 1 \\
-1 & 0
\end{array}\right| \cdot\left(\begin{array}{c}
\beta_{1 i} \\
\beta_{2 i}
\end{array}\right) \text {. }
$$

As in (2.6) let

Solving (3.6) for $\beta_{j i}(j=1,2)$ we get

$$
\left|\begin{array}{ll}
a_{i} & b_{i} \\
c_{i} & d_{i}
\end{array}\right|=\prod_{j=1}^{i-1}\left|\begin{array}{cc}
\bar{d}_{i-j} & 1 \\
-1 & 0
\end{array}\right| .
$$

$$
\left(\begin{array}{c}
\beta_{1 i} \\
\beta_{2 i}
\end{array}\right)=\left|\begin{array}{cc}
d_{i} & -b_{i} \\
-c_{i} & a_{i}
\end{array}\right| \cdot\left(\begin{array}{l}
0 \\
1
\end{array}\right)=\left(\begin{array}{c}
-b_{i} \\
a_{i}
\end{array}\right) .
$$

Now we can write down $\partial_{2}$ :

$$
\partial_{2}=\left(\alpha_{j i}\right)=\left|\begin{array}{cccc}
0 & 0 & -b_{2} & -b_{3} \\
0 & 1 & a_{2} & a_{3}
\end{array}\right|: \mathbf{Z}\left\langle h_{2}^{0}, h_{2}^{1}, h_{2}^{2}, h_{2}^{3}\right\rangle \rightarrow \mathbf{Z}\left\langle h_{1}^{1}, h_{1}^{2}\right\rangle .
$$

Finally, recall that $H_{2}(A, \mathbf{Z})=\operatorname{ker} \partial_{2}$. There are at least two independent elements in the kernel; we may take them as $h_{2}^{0}$ and $p_{1} h_{2}^{1}+p_{2} h_{2}^{2}+p_{3} h_{2}^{3}$, where the vector $\left(p_{i}\right)$ is the same as the one used in constructing the parameter space M. As alluded to in the remark following that construction, we now prove that these two generate $\mathrm{H}_{2}$. 
(3.8) Lemma. $\operatorname{rank} H_{2}(A, \mathbf{Z})=2$ and $\operatorname{rank} H_{1}(A, \mathbf{Z})=0$.

Proof. Obviously, rank $H_{2} \geq 2$, and equality implies the second statement too. By the long exact sequence for the pair $(Y, A)$ we have (following Looijenga $[9,5.1])$

$$
\begin{array}{cc}
H_{3}(Y) & \rightarrow H_{3}(Y, A) \rightarrow H_{2}(A) \stackrel{i_{*}}{\rightarrow} H_{2}(Y) \rightarrow \cdots \\
0 & H^{1}(D)
\end{array}
$$

where we identify $H_{3}(Y, A)$ with $H^{1}(D)$ by (relative) Poincaré duality and $i: A \rightarrow Y$ is the inclusion. The image of $i_{*}$ is contained in the orthogonal complement of the lattice spanned by the components of $D$, and the kernel of $i_{*}$ is of rank one. We claim that the image of $i_{*}$ is at most of rank one; it then follows from our remarks above that this rank is exactly one, finishing the argument. Recall that $Y$ is obtained by blowing up a triangle of lines in $P^{2}$ at vertices followed by three internal blowups. There must be $n-3$ corner blowups to produce a cycle of length $n$, followed by three interior blowups, for a total of $n$ blowups. Therefore $H_{2}(Y)$ has rank $n+1$ : the $n$ exceptional curves and the class of a line are generators. Since the span of the components of $D$ is a (negative) definite lattice on $n$ generators, its orthogonal complement must have rank one.

Now we can compute the monodromy on $H_{2}(A, \mathbf{Z})$. Recall that $Y_{t}$ is obtained by blowing up $\bar{Y}$ at three (interior) points on $\bar{D}$ given in the coordinates of the components as $\left(t^{e_{1}}, t^{e_{2}}, t^{e_{3}}\right)$.

(3.9) Theorem. Let $x \in H_{2}\left(Y_{t} \backslash D, \mathbf{Z}\right)$ be written in terms of the handle-body decomposition as $x=\sum_{i=0}^{p} m_{i} h_{2}^{i}$. Let $m=\sum_{i=1}^{p} m_{i} e_{i}$, where $e_{i}=$ order of $S_{i}$ regarded as a map from $\mathbf{M}$ to $\bar{D}_{i}$.

Then the monodromy action on $x$ is $x \rightarrow x+m f$, where $f$ is the class of the fiber of $\tau$.

Notes. (1). The handle-body representation of $f$ is just $h_{2}^{0}$. In particular $f$ is unaffected by monodromy.

(2) For the two generators described above, i.e., $f=h_{2}^{0}$ and $q=p_{1} h_{2}^{1}+$ $p_{2} h_{2}^{2}+p_{3} h_{2}^{3}$, we have $q \rightarrow q+f$ and $f \rightarrow f$ under the monodromy.

(3) For purposes of computing monodromies we restrict the family $\mathbf{Y} \rightarrow$ $\mathbf{M} \approx \mathbf{C}^{*}$ to the unit circle.

Proof. The calculation is performed locally near a component of $\bar{D}$. We will count the contribution of each handle-body. Let $p$ be one of the blowup points, $m$ the multiplicity of the corresponding handle-body in $q$. As $t$ moves around the origin in $\mathbf{M}=\mathbf{C}^{*}, p=t^{e_{i}}$ moves around $0 \in \bar{D}_{i}\left|e_{i}\right|$ times, where the sign of $e_{i}$ determines the direction. For simplicity suppose that $e_{i}=1$. Let $\varepsilon$ be the loop in $\bar{D}_{i}^{*}$ traversed by $p$. Let $N^{\prime}(\varepsilon) \subset N(\varepsilon)$ be tubular neighborhoods of $\varepsilon$ in $\bar{Y}$ chosen so that the radius of $N(\varepsilon)$ is strictly greater than that of $N^{\prime}(\varepsilon)$ and so that $N^{\prime}(\varepsilon) \cap \bar{A} \subset \bar{\tau}$. A cross section is drawn in Figure 5 . 


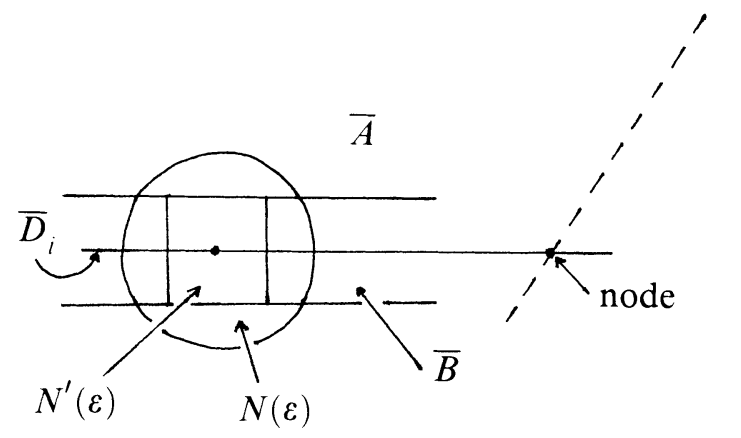

Figure 5

There exists an isotopy $\phi: \bar{Y} \times[0,1] \rightarrow \bar{Y}$ of $\bar{Y}$ which is the identity (for fixed $s \in[0,1])$ outside $N(\varepsilon)$, and which at time $s$ rotates $N^{\prime}(\varepsilon)$ by $2 \pi s . \phi_{1}$ is then the identity on $N^{\prime}(\varepsilon)$ as well. If $N$ is a neighborhood of $p$ contained in $N^{\prime}(\varepsilon)$, we can remove $N$ and attach the handles of $A$ and $B$, and extend $\phi_{1}$ to a nontrivial diffeomorphism of $Y_{t}$. The core of the 2-handle attached to $\bar{A}$ must pass through $N(\varepsilon) \backslash N^{\prime}(\varepsilon)$ to connect with the other 2-handles to form $q$; it now traces out a copy of $f$ before leaving $N^{\prime}(\varepsilon)$. Finally, if the 2-handle has a multiplicity $m$, then $m$ copies of the core are used to construct $q$, and each one traces out a copy of $f$. It is clear that an $e_{i}$ other than 1 effects the contribution of $h_{2}^{i}$ by multiplication.

Remark. This proof will also be used to compute the monodromy on $H_{1}(\tau, \mathbf{Z})$.

We are now ready to prove that the family $\mathbf{Y} \stackrel{\pi}{\rightarrow} \mathbf{M}$ is in fact a universal deformation space (in the sense of Looijenga) for each of its members.

(3.10) Theorem. The family $\pi: \mathbf{Y} \rightarrow \mathbf{M}$ is a universal deformation space of the pair $\left(Y_{t}, D_{t}\right)$ for each $t \in \mathbf{M}$; i.e., for each $t, \pi$ induces an isomorphism $\rho_{\pi, t}: T_{t} \mathbf{M} \rightarrow H^{1}\left(\boldsymbol{\theta}_{Y}(\log D)\right)$ where $\boldsymbol{\theta}_{Y}(\log D)$ is the sheaf of analytic vector fields on $Y$ preserving $D, T_{t} \mathbf{M}$ is the tangent space to $\mathbf{M}$ at $t$, and $\rho_{\pi, t}$ is the Kodaira-Spencer homomorphism.

Proof. Following Looijenga, we need only show that the period map is a local isomorphism: Let $w_{t}$ be a meromorphic two-form on $Y_{t}$ satisfying $\operatorname{Div}\left(w_{t}\right)=$ $-D_{t}$; this determines $w_{t}$ up to a nonzero scaler. In local coordinates we can write $w_{t}$ as $w_{t}=d x \wedge d y / x y$. Locally near $t_{0} \in \mathbf{M}$ we can identify $H_{2}\left(Y_{t} \backslash D ; \mathbf{C}\right)$ with $\mathbf{C}^{2}$. Then the following map is well defined.

Definition. $\phi(t)=\left[w_{t}\right] \in \mathbf{P} H^{2}\left(Y_{t} \backslash D ; \mathbf{C}\right)$ is the period map associated to the family $\pi: \mathbf{Y} \rightarrow \mathbf{M}$, where $\left[w_{t}\right]$ is the equivalence-class of (the class of) $w_{t}$ mod scalars.

We will consider a slightly different version of $\phi$ : Let

$$
\mu(t)=\exp 2 \pi i\left(\int_{q} w_{t} / \int_{f} w_{t}\right) .
$$


As shown in [9], $\int_{f} w_{t}$ is just the double residue of $w_{t}$ at a node of $D$ (multiplied by $(2 \pi i)^{2}$ ), which is always nonzero. Dividing by it normalizes $w_{t}$ locally. Since $\mu$ factors through $\phi$, it suffices to show that $\mu$ is a local isomorphism.

Claim. $\mu(t)=c t$ where $c$ is a nonzero constant.

Proof. The calculation imitates the proof of Proposition 5.2 in [9]. We must sketch the model for $q$ that we use in evaluating the integral of $w_{t}$. As above, we regard $Y_{t}$ as being obtained from $\bar{Y}$ by excising neighborhoods of points on $\bar{D}$ and attaching handles. Briefly, $q$ is composed of three "pieces":

Let $E_{i}$ be the exceptional curve meeting $D_{i}$ at the point $z_{i}=t^{e_{i}}$, where $z_{i}$ is the affine coordinate introduced in the description of $\mathbf{M}$.

Let $\gamma_{i}$ be a path from $t^{e_{i}}$ to $z_{i}=1$ on $\bar{D}_{i}$ which avoids $z_{i}=0$ and $z_{i}=\infty$. Let $\delta_{i}$ be a tubular neighborhood of $D_{i}$ so that $E_{i} \cap \delta_{i}$ is a fiber of $\delta_{i}$. Then $q$ can be realized as the union of

(1) $p_{i}$ copies of $E_{i} \backslash\left(E_{i} \cap \delta_{i}\right)$;

(2) $p_{i}$ copies of $\left.\partial \delta_{i}\right|_{\gamma_{i}}$;

(3) A cell complex lying within $\bar{Y} \backslash N(\bar{D})$ which remains fixed as $t$ moves.

The integral of the $w_{t}$ over the pieces in (1) is zero, since these pieces are analytically embedded complex disks and $w_{t}$ is a holomorphic two-form. The integral over the complex in (3) is constant in $t$, since this portion of the model of $q$ can be held fixed while $t$ moves. We are left with computing the integral over the tubes $\left.\partial \delta_{i}\right|_{\gamma_{i}}$. As in [9],

$$
\begin{aligned}
\int_{\left.\partial \delta_{i}\right|_{\gamma_{i}}} w_{t} & =p_{i} \int_{\gamma_{i}} 2 \pi i \operatorname{Res}\left(w_{t}\right)=p_{i} \int_{\gamma_{i}}(2 \pi i)^{-1} x_{i}^{-1} d x_{i} \\
& =\left.p_{i}(2 \pi i)^{-1} \ln \left(x_{i}\right)\right|_{z_{i}=1} ^{z_{i}=t^{e_{i}}}=p_{i} \cdot(2 \pi i)^{-1} \ln \left(t^{e_{i}}\right) .
\end{aligned}
$$

Then $\int_{q} w_{t}=\sum_{i=1}^{3} p_{i}(2 \pi i)^{-1} \ln \left(t^{e_{i}}\right)+C$, so

$$
\mu(t)=\exp 2 \pi i \int_{q} w_{t}=(t)^{\sum e_{i} p_{i}} \cdot e^{2 \pi i C}=c \cdot t
$$

(since $\left.\sum e_{i} p_{i}=1\right)$.

Next we quote Pinkham's calculation of the automorphism group of the Inoue surface $X$. Recall the number theoretic construction of $X$. Let $\alpha$ be a generator of $U^{+}$, and denote by $\bar{M}$ the module $(\alpha-1)^{-1} M$. It is easy to see that $U(\bar{M})=U(M)$, so we can form $G(\bar{M}, U)$.

(3.11) Theorem (Pinkham). $G(\bar{M}, U) / G\left(\dot{M}, U^{+}\right)$is the full group of complex automorphisms of $X$.

It follows from the proof of the theorem how the automorphism group acts on $H_{1}(\tau, \mathbf{Z})$, where $\tau$ sits in $X$, as it does in $Y_{t}$, as the boundary of a regular neighborhood of $D$. In fact, it follows from the (second) construction of $X$ 
that $X \backslash(D, C) \approx \mathbf{R} \times \tau$ (topologically). On the other hand, $X \backslash(D, C) \approx$ $\mathbf{H} \times \mathbf{C} / G\left(M, U^{+}\right)$, so

$$
\begin{aligned}
\pi_{1}(\tau) & =\pi_{1}(\mathbf{R} \times \tau)=G\left(M, U^{+}\right), \\
H_{1}(\tau, \mathbf{Z}) & =G\left(M, U^{+}\right) / G^{\prime}\left(M, U^{+}\right),
\end{aligned}
$$

where $G^{\prime}\left(M, U^{+}\right)$is the commutator subgroup of $G\left(M, U^{+}\right)$.

Then the action of the automorphism group of $X$ acts on $H_{1}(\tau, \mathbf{Z})$ because every automorphism restricts to an automorphism of $X \backslash(D, C)$, and the action is given by conjugating $G\left(M, U^{+}\right)$by elements of $G(\bar{M}, U)$; this action descends to the quotients. There is the following explicit formula:

$$
(u, \bar{m}):(v, m) \rightarrow(v, u m+(1-v) \bar{m})
$$

where $(u, \bar{m}) \in G(\bar{M}, U),(v, m) \in G\left(M, U^{+}\right)$.

It is interesting to note that this action is not well defined on $G\left(M, U^{+}\right)=$ $\pi_{1}(\tau)$; i.e., $G(\bar{M}, U) / G\left(M, U^{+}\right)$does not act on $G\left(M, U^{+}\right)$. This reflects the fact that automorphisms of $X$ do not preserve a base point on $\tau$.

Finally, we should mention that we have skipped over one point: we have assumed that the cycles $C$ and $D$ are reduced in the sense that the sequences $\left[c_{1}, \ldots, c_{m}\right]$ and $\left[d_{1}, \ldots, d_{n}\right]$ are not repetitions of smaller sequences, e.g., $[2,3,2,3]$. This corresponds to replacing $U^{+}$by a subgroup $V$ in the construction of $X$. Then the automorphisms of $X$ are of the form

$$
G(\bar{M}, U) / G(M, V) \text {. }
$$

Assuming (as we have) that $V=U^{+}=U, G(\bar{M}, U) / G\left(M, U^{+}\right)$is just isomorphic to $\bar{M} / M$. In general, $\bar{M} / M$ is the kernel of the induced action on $H_{2}(X, \mathbf{Z})$. That is, $\bar{M} / M$ fixes the components of $C$ and $D$. The cokernel essentially divides $C$ and $D$ by their periods, giving the reduced cycles; and if $U^{+} \neq U$, we are in the self-dual case $(C=D)$, and $U^{+} / U$ is the involution on $X$. We are mainly concerned with the action of $\bar{M} / M$, so we will assume $U=U^{+}=V$. We can take $(1, \bar{c}), \bar{c} \in \bar{M}=(\alpha-1)^{-1} M$, as a generator of $G(\bar{M}, U) / G(M, U) .(1, \bar{c})$ acts on $G:=G(M, U)$ as an element of $\bar{G}:=G(\bar{M}, U)$ by $\left(\alpha^{n}, m\right) \rightarrow\left(\alpha^{n}, m+\left(1-\alpha^{n}\right) \bar{c}\right)$, where $\alpha$ generates $U$, and $m \in M$. Write $\bar{c}=c /(1-\alpha), c \in M$. Then the formula becomes

$$
\left(\alpha^{n}, m\right) \rightarrow\left(\alpha^{n}, m+\left(1-\alpha^{n}\right) c /(1-\alpha)\right)=\left(\alpha^{n}, m+\left(1+\alpha+\cdots+\alpha^{n-1}\right) c\right) .
$$

But in homology, $\alpha c=c$ : this is because we may identify $M$ with $H_{1}(f, \mathbf{Z})$, $f=$ fiber of $\tau$, and multiplication by $\alpha$ is then just the monodromy of $\tau$ as a $T^{2}$-bundle over $S^{1}$. Therefore $\left(\alpha^{n}, m+\left(1+\alpha+\cdots+\alpha^{n-1}\right) c\right)=\left(\alpha^{n}, m+n c\right)$ in $H_{1}(\tau, \mathbf{Z})$; thus, $\left(\alpha^{n}, m\right) \rightarrow\left(\alpha^{n}, m+n c\right)$ is the action of $(1, \bar{c}) \in \bar{M} / M$ on $H_{1}(\tau, \mathbf{Z})$.

Note that the exponent of $\alpha$ in $\left(\alpha^{n}, m\right)$ can be interpreted as the "sectional component" of $\left(\alpha^{n}, m\right) \in H_{1}(\tau, \mathbf{Z})$. We can factor $H_{1}(\tau, \mathbf{Z})$ into free and torsion parts; then the sections of $\tau$ generate the free part and the image $i_{*} H_{1}(f, \mathbf{Z})$ is the torsion part, where $i: f \rightarrow \tau$ is inclusion. Explicitly, 
$H_{1}(\tau, \mathbf{Z}) \approx \mathbf{Z} \oplus$ (torsion); the map $\left(\alpha^{n}, m\right) \rightarrow n$ is projection on the first factor, and (torsion) can be identified with $M /(\alpha-1) M$. (This description relies on the negative-definiteness of $D$.) The action can finally be written as

$$
x \rightarrow x+(x f) c
$$

where $x \in H_{1}(\tau, \mathbf{Z}),(x f)$ is the intersection of $x$ and the fiber $f$, and $c$ is the image of $\bar{c} \in \bar{M} / M \rightarrow M /(\alpha-1) M$ under multiplication by $(\alpha-1)$.

(3.13) Theorem. The monodromy action on $H_{1}(\tau, \mathbf{Z})$, where we regard $\tau$ as a subspace of $Y_{z}$, is given by the formula

$$
x \rightarrow x+(x f) c .
$$

Remarks. (1) The proof will describe the cycle $c \in$ tor $H_{1}(\tau, \mathbf{Z})$ explicitly.

(2) There is an obvious similarity between the formula and Picard-Lefschetz transformations. We will show that in fact the monodromy is a kind of Dehntwist on $\tau$, along a flow defined by $c$.

(3.14) Corollary. The family of neighborhoods $N\left(D_{t}\right) \subset Y_{t}, t \in \mathbf{M}$, becomes topologically trivial after a finite base change.

Proof. Let $n$ be the order of tor $H_{1}(\tau, \mathbf{Z})$. Because $c$ is a torsion class, and $(c f)=0$, iteration of the formula $n$ times gives the identity. Thus base change of degree $n$ gives a family in which the monodromy on $H_{1}(\tau, \mathbf{Z})$ is trivial. Therefore the monodromy on $\tau$ itself is isotopic to the identity, and the family over $\mathbf{M}$ is trivial.

Proof of (3.13). First we will describe an action on $\tau$ and then show using the constructions of Theorem (3.9) that it is induced by the monodromy. Choose an interval $I \approx[0,1]$ on $S^{1}$, where $S^{1}$ is the base of the fibration $\tau \rightarrow S^{1}$. Trivialize $\tau$ over $I$ so that $\left.\tau\right|_{I} \approx f \times I, f=2$-torus. There is an isotopy on $f$ which begins and ends with the identity and at time $t \in[0,1$ is translation by $2 \pi t$ along the cycle $C$. (Here $C$ is any primitive 1-cycle on $f$ (see Figure 6).) This gives a map $g:\left.\left.\tau\right|_{x} \rightarrow \tau\right|_{x}$ which is the identity at the "ends," $\left.\tau\right|_{0}$ and $\left.\tau\right|_{1}$. Patch this map to the identity on $\tau \mid S^{1} \backslash I$, to get a map $\tau \rightarrow \tau$. (This may be compared to a Dehn-twist on a real surface. Let $C$ be the twist cycle; a neighborhood of $C$ is isomorphic to $C \times I$. The twist is obtained by isotoping $C$ through a complete rotation in $C \times I$ and patching the ends to the identity on the rest of the surface (Figure 7).) Notice what happens to a section of $\tau$ under the above map: it gets twisted by the cycle which defines the isotopy (Figure 8). On the other hand, any torsion cycle is supported on a fiber, which we may choose away from the site of the action of $g$, and so is unaffected. This agrees with the formula.

Now for the proof (and the description of $c$ ). Recall the description of the monodromy on $Y_{t} \backslash D$ in Theorem (3.9). For simplicity we will assume that only one of the sections $S_{i}: \mathbf{M} \rightarrow \bar{Y}$ is varying and that its order is equal to one; the effect of more varying sections or other orders is linear. As before, 


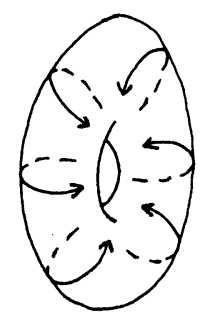

FIGURE 6
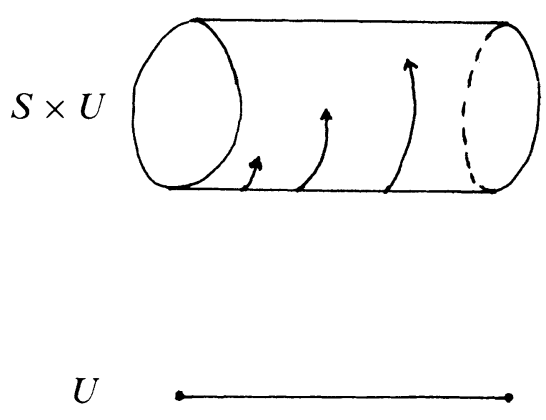

FIGURE 7

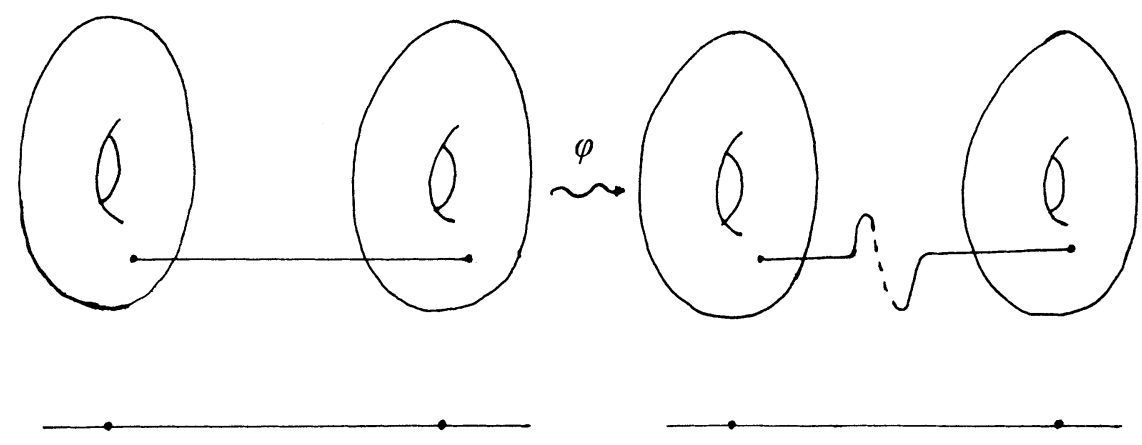

FIGURE 8

divide $\bar{Y}$ into $\bar{A}$ and $\bar{B}$. Previously we focused on the effect of monodromy on $A$ (the union of $\bar{A}$ and 2-handles); now we will focus on $\bar{B}$ and $B$. Recall that we first perform an isotopy on $\bar{Y}$ (a homotopically trivial action) and then cut out a neighborhood of a fixed point and attach handles, finally extending the isotopy over the handles to obtain a diffeomorphism. Consider now the isotopy restricted to $\bar{B}$. It induces an action (trivial at this point) on $\bar{\tau}=\partial \bar{B}$. Specifically, the path $\varepsilon$ traced by the section $S$ lifts to a loop in $\bar{\tau}$ which is 


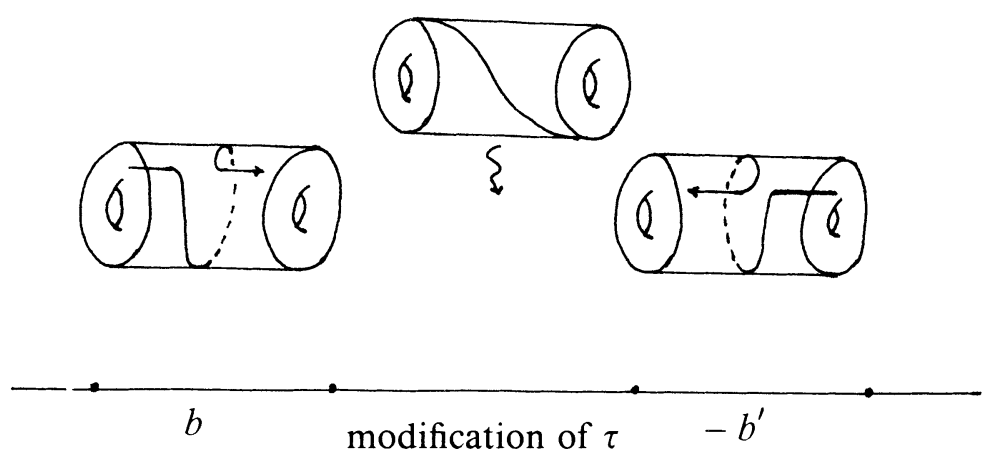

FIGURE 9

supported on a fiber. (As before we restrict $\mathbf{Y} \rightarrow \mathbf{M}$ to $S^{1}$, the unit circles in $C^{*}$.) The intersection of $\bar{\tau}$ and the locus of the action of the isotopy is just the restriction of $\bar{\tau}$ to an interval, over which $\bar{\tau}$ is naturally trivialized by the local coordinates of $\bar{Y}$. Let $b$ be the loop which $\varepsilon$ lifts to. In local coordinates $(x, y), b$ is part of a natural basis: $a=\left[\left(r, s e^{i \phi}\right) \mid \phi \in[0,2 \pi)\right]$, $b=\left[\left(r e^{i \theta}, s\right) \mid \theta \in[0,2 \pi)\right] \quad\left(r, s\right.$ fixed). The action of the isotopy on $\left.\bar{\tau}\right|_{I}$ divides into three pieces: on the first third of $I$, it twists $\bar{\tau}$ by one full rotation along $b$; for the second third it is the identity; and on the last third it twists $\bar{\tau}$ by one full rotation along $-b$. Now, however, we must alter $\bar{B}$ and $\bar{\tau}=\partial \bar{B}$ : we remove $N(p)$ and glue in a new 2-handle. Notice that the (explicit) cycles we twist $\bar{\tau}$ by, $b$ and $-b$, lie on opposite sides of the interval of $\bar{\tau}$ that is modified; the effect is that these cycles no longer add up to zero (Figure 9). The natural basis that we had for $\bar{\tau}$ is no longer unique: it depends on which side of the interval over which $\bar{\tau}$ is modified that we take them on. If $\{a, b\}$ is the natural basis on one side of the action and $\left\{a^{\prime}, b^{\prime}\right\}$ is the same basis on the other side (before modification), then after modification the relationship between them is given by the matrix $\left|\begin{array}{cc}1 & -1 \\ 0 & 1\end{array}\right|$. Explicitly, $a^{\prime}=a$ and $b^{\prime}=b-a$. In the basis $\{a, b\}$, the twisting of $\tau$ induced by monodromy is along the cycle $b-b^{\prime}=b-(b-1)=a$. This is the cycle $c$ in (3.12).

\section{4}

In this section we collect an assortment of numerical consequences of our work. Assume $(Y, D)$ is a rational pair with a one-dimensional moduli space. Then, as we saw before, $(Y, D)$ dominates a toroidal surface $(\bar{Y}, \bar{D})$, and exactly three interior blowups on $\bar{D}$ are required to obtain $(Y, D)$. Let $E_{i}$ $(i=1,2,3)$ be the exceptional curves to these. Then $\operatorname{Pic}(Y)$ is generated (redundantly) by the $E_{i}$ and the components of $D$. Let $\left\langle D_{j}\right\rangle$ be the span of the components of $D$ and let $Q$ be the orthogonal complement in $H_{2}\left(Y_{t} ; \mathbf{Z}\right)$ of $\left\langle D_{j}\right\rangle$. By the proof of Lemma (3.7), rank $Q=1$. Let $q$ be a generator of 
$Q$; write $q$ in the generators $\left\langle E_{i}, D_{j}\right\rangle$ :

$$
q=\sum a_{i} E_{i}+\sum b_{j} D_{j} .
$$

(4.2) Definition. $p_{i}=a_{i}-b_{i}$ (where we label the $D_{j}$ so that $\left.D_{i} E_{i}=1\right)$.

(4.3) Lemma. The vector $\left(p_{i}\right)$ is determined up to \pm 1 .

Proof. The expression for $q$ is unique up to the radical of $\left\langle E_{i}, D_{j}\right\rangle$ and the fact that $-q$ also generaters $Q_{\mathbf{Z}}$.

$$
\begin{aligned}
& \text { Suppose } & x & =\sum \bar{a}_{i} E_{i}+\sum \bar{b}_{j} D_{j} \in \operatorname{rad}\left\langle E_{i}, D_{j}\right\rangle . \\
& \text { Then } & x E_{i} & =0, \quad i=1,2,3, \\
& \text { implies } & x E_{i} & =-\bar{a}_{i}+\bar{b}_{i}=0, \\
& \text { so } & \bar{b}_{i} & =\bar{a}_{i} .
\end{aligned}
$$

Therefore $x$ can be written as $x=\sum \bar{b}_{i}\left(D_{i}+E_{i}\right)+\sum_{j \notin\{i\}} \bar{b}_{j} D_{j}$. Adding an expression of this form to $q$ does not change $\left(p_{i}\right)$.

(4.4) Lemma. If $x=\sum \bar{b}_{i}\left(D_{i}+E_{i}\right)+\sum \bar{b}_{j} D_{j} \in \operatorname{rad}\left\langle E_{i}, D_{j}\right\rangle$ then $\left(p_{i}\right)\left(\bar{b}_{i}\right)=0$. Proof.

$$
\begin{aligned}
q x & =q\left(\sum \bar{b}_{i}\left(D_{i}+E_{i}\right)+\sum \bar{b}_{j} D_{j}\right), \\
0 & \left.=q\left(\sum \bar{b}_{i} E_{i}\right) \quad \text { (because } q D_{j}=0, \text { all } j\right), \\
0 & =\sum p_{i} \bar{b}_{i} \cdot \quad \square
\end{aligned}
$$

Let $M=\left(D_{i} \cdot D_{j}\right)$ be the intersection matrix of the cycle $D$, and let $\bar{I}$ be the diagonal matrix with $j$ th entry equal to one if $\bar{D}_{j}$ has received an interior blowup and zero otherwise. Let $\bar{M}=M+\bar{I} ; \bar{M}$ is just the intersection matrix for the cycle $\bar{D}$.

(4.5) Lemma. Let $\left.M^{-1}\right|_{3 \times 3}$ be the $3 \times 3$ matrix of nonzero entries of $\bar{I}\left(M^{-1}\right) \bar{I}$. $\left.M^{-1}\right|_{3 \times 3}$ has three rational eigenvalues; -1 is a doubleroot of its characteristic equation, and the vector $\left(p_{i}\right)$ is an eigenvector for the other eigenvalue. The matrix $\left.M^{-1}\right|_{3 \times 3}+I$ may be written

$$
\left|\begin{array}{l}
\mu_{1}\left(p_{1}, p_{2}, p_{3}\right) \\
\mu_{2}\left(p_{1}, p_{2}, p_{3}\right) \\
\mu_{3}\left(p_{1}, p_{2}, p_{3}\right)
\end{array}\right|
$$

where

$$
\begin{aligned}
\mu_{i} & =p_{i}(\mu+1) /\left(p_{i}\right)\left(p_{i}\right), \\
\mu & =\left.\operatorname{det} M^{-1}\right|_{3 \times 3}=\text { eigenvalue for }\left(p_{i}\right) .
\end{aligned}
$$

Proof. Clearly $\operatorname{rank}\left(\operatorname{rad}\left\langle E_{i}, D_{j}\right\rangle\right)=2$, since $\operatorname{rank} \operatorname{Pic}(Y)$ is one more than the number $n$ of components of $D$, and $\left\langle E_{i}, D_{j}\right\rangle$ contains $n+3$ generators. The 
general element of the radical has the form $x=\sum \bar{b}_{i}\left(D_{i}+E_{i}\right)+\sum_{j \notin\{i\}} \bar{b}_{j} D_{j}$. The conditions $x D_{k}=0$ reduce to the system

$$
\bar{M}\left(\bar{b}_{j}\right)=0 \text {. }
$$

Therefore $\bar{M}$ has a rank-two kernel. Write this in terms of $M$ :

$$
(M+\bar{I})\left(\bar{b}_{j}\right)=0 \text {. }
$$

Multiply by $M^{-1}$ :

$$
\left(I+M^{-1} \bar{I}\right)\left(\bar{b}_{j}\right)=0, \quad\left(\bar{b}_{j}\right)+M^{-1} \bar{I}\left(\bar{b}_{j}\right)=0 .
$$

Restrict this to the rows which are nonzero in $\bar{I}$ :

$$
\left(\bar{b}_{i}\right)+\left.M^{-1}\right|_{3 \times 3}\left(\bar{b}_{i}\right)=0, \quad\left(\bar{b}_{i}\right) \text { are now 3-tuples, }
$$

or

$$
\left(\left.M^{-1}\right|_{3 \times 3}+I\right)\left(\bar{b}_{i}\right)=0, \quad I=\text { rank } 3 \text { identity matrix } .
$$

This system must have two independent solutions. (Otherwise there exists $x \in \operatorname{rad}\left\langle E_{i}, D_{j}\right\rangle$ with coefficients of $E_{i}$ : equal to zero; i.e., $x$ is in the span of the $D_{j}$-but the $D_{j}$ span a negative-definite lattice.) Therefore -1 is a double eigenvalue. This implies that $\mu:=\left.\operatorname{det} M^{-1}\right|_{3 \times 3}$ is the third eigenvalue.

Let

$$
\left.M^{-1}\right|_{3 \times 3}+I=\left|\begin{array}{c}
\mathbf{v}_{1} \\
\mathbf{v}_{2} \\
\mathbf{v}_{3}
\end{array}\right|, \quad \mathbf{v}_{i}=\text { row vector } .
$$

Since -1 is a double eigenvalue, all $\mathbf{v}_{i}$ are proportional. Let $\mathbf{v}=\mathbf{Z}$-primitive vector in the span of the $\mathbf{v}_{i}$, and define $\mu_{i}$ by $\mathbf{v}_{i}=\mu_{i} \mathbf{v}$. Write $\mathbf{v}=\left(v_{1}, v_{2}, v_{3}\right)$. Then since $\left.M^{-1}\right|_{3 \times 3}+I$ is symmetric, $\mu_{i} v_{j}=\mu_{j} v_{i}$. Therefore the vector $\left(\mu_{i}\right)$ is proportional to $\left(v_{i}\right)$. It is easy to calculate the constant of proportionality via the determinant of $\left.M^{-1}\right|_{3 \times 3}$; it is just $(\mu+1) /\left(v_{i}\right)\left(v_{i}\right)$.

Finally, (4.6) implies that $\left(v_{i}\right)\left(b_{i}\right)=0$ for any $x=\sum \bar{b}_{i}\left(E_{i}+D_{i}\right)+\sum \bar{b}_{j} D_{j} \in$ $\operatorname{rad}\left\langle E_{i}, D_{j}\right\rangle$. By Lemma 4.4 we must have $\left(v_{i}\right)=\left(p_{i}\right)$.

(4.7) Lemma. Two formulas for $q^{2}$ :

(1) $q^{2}=-\sum p_{i}\left(p_{i}+b_{i}\right)$ in the notation of (4.2).

(2) $q^{2}=m\left|p_{1} p_{2} p_{3}\right|-\sum p_{i}^{2}, \quad m=\operatorname{lcd}\left(C_{i} C_{j}\right) ; C_{i}=$ attaching circles for 2-handles.

Proof. For (1), square the expression for $q$ in (4.1), using the fact that $q \cdot D_{j}=0$ for all $j$, and then substitute the $p$, using $a_{i}=p_{i}+b_{i}$.

For (2), recall the handle-body decomposition of $Y$ and $\bar{Y}$; we must add three 2-handles to $\bar{Y} \backslash N(\bar{D})$ to get $Y \backslash N(D)$. $q$ is expressed as a sum of these handles.

We claim that the coefficients are just the $p_{i}$. Let $C_{i}$ be the attaching circle of $h_{z}^{i}, i=1,2,3$. If we write $C_{i}=\left(a_{i}, b_{i}\right)$ in some basis of $H_{1}(\bar{f}, Z)$, 
$\bar{f}=$ fiber of $\bar{\tau}$, it can be shown that $\left(a_{i}\right)$ and $\left(b_{i}\right)$ are the coefficients of $\left(E_{i}+D_{i}\right)$ for independent elements of $\operatorname{rad}\left\langle E_{i}, D_{j}\right\rangle$. On the other hand, if $q=\sum q_{i} h_{2}^{i}$, then $\sum q_{i} a_{i}=\sum q_{i} b_{i}=0$ by (3.7). By Lemma $4.4\left(q_{i}\right)=\left(p_{i}\right)$.

Now we can compute $q^{2}$. A model for $q$ is constructed by taking $p_{i}$ parallel copies of the core of $h_{2}^{i}, i=1,2,3$, and tubing their boundaries together inside $\bar{Y} \backslash N(\bar{D})$. Each boundary is a 1-cycle on a fiber of $\bar{f}$; if we move a core sheet, it must intersect itself and all the others $(-1)$ times, if we want to move its boundary parallel to itself. (This is because the attaching degree is -1 ; see (3.2).) Therefore each handle contributes $-p_{i}^{2}$ to $q^{2}$. Next we must move the tubing. We may think of the tubes from each handle being drawn back to a central 2-torus inside $\bar{A}$, which is just $T^{2} \times B^{2}$. (To connect the tubes using only pieces of a 2-torus, we will only be able to build a cell complex, not a manifold.) We can move the tubes from two of the handles off to a different central 2-torus without meeting the old tubing. But the third set of tubing (from the remaining handle) must cross the path of the original tubing from one of the other handles. If $h_{2}^{i}$ and $h_{2}^{j}$ are the handles involved, the intersection number is $\left(C_{i} \cdot C_{i}\right)$ for each pair of tubes; therefore the total contribution of the tubes is $\left(p_{i} \cdot C_{i}\right)\left(p_{j} C_{j}\right)$, counted positively. Let $x_{k}=C_{*} C_{* *}$, where $\{*, * *\}=\{1,2,3\} \backslash\{k\}$. (Also, put a cyclic ordering on $\{1,2,3\}$, and require $(*, * *)$ to be consecutive. This takes the antisymmetry of the pairing into account.) Then $\left(x_{k}\right)$ is proportional to $\left(p_{i}\right):$ If $C_{i}=\left(a_{i}, b_{i}\right)$ then $C_{i} C_{j}=\left(a_{i} b_{j}-b_{i} a_{j}\right.$ ) (up to \pm ); now compute $\sum x_{i} C_{i}$ and see that it is zero. By Lemma 4.4 and the above, $\left(x_{i}\right)$ is proportional to $\left(p_{i}\right)$. Since $\left(p_{i}\right)$ is primitive, $\left(x_{i}\right)=m\left(p_{i}\right)$ where $m=\operatorname{lcd}\left\{C_{i} C_{j}\right\}$. Therefore the intersection number of the tubes is equal to

$$
\begin{aligned}
\left(p_{i} C_{i}\right) \cdot\left(p_{j} C_{j}\right) & =p_{i} p_{j} C_{i} C_{j}=p_{i} p_{j} m p_{k}, \quad\{k\}=\{1,2,3\} \backslash\{i, j\}, \\
& =m p_{1} p_{2} p_{3} .
\end{aligned}
$$

Remarks. The following are equivalent:

(1) $m=1$.

(2) The Milnor fiber is simply connected.

(3) The lattice $\left\langle D_{j}\right\rangle$ generated by the components of $D$ is primitively embedded in $\operatorname{Pic}(Y)$.

Example. Take $D:=[2, \ldots, 2,3]$ (nine 2's). Then

$$
-D^{2}=1
$$

and

$$
r=\text { number of components }=10 .
$$

$D$ sits on a rational surface: Blowup $P^{2}$ on a nodal cubic nine times, and make one interior blowup. Label $D_{i}$ consecutively ending with the -3 component. Then we can blow down on components 2,5 , and 9 to get a toroidal surface. 
We have $\left(p_{i}\right)=(4,5,3)$,

$$
\begin{gathered}
\left.M^{-1}\right|_{3 \times 3}=\left|\begin{array}{lll}
-2.6 & -2.0 & -1.2 \\
-2.0 & -3.5 & -1.5 \\
-1.2 & -1.5 & -1.9
\end{array}\right|, \\
\left.M^{-1}\right|_{3 \times 3}+I=\left|\begin{array}{l}
-4 / 10 \cdot(4,5,3) \\
-5 / 10 \cdot(4,5,3) \\
-3 / 10 \cdot(4,5,3)
\end{array}\right| .
\end{gathered}
$$

The cycle is primitively embedded. Therefore

$$
\begin{aligned}
q^{2} & =p_{1} p_{2} p_{3}-\sum p_{i}^{2} \\
& =4 \cdot 5 \cdot 3-(16+25+9) \\
& =60-50=10 .
\end{aligned}
$$

\section{REFERENCES}

1. R. Friedman, preprint.

2. _ Thesis, Harvard University, 1981.

3. R. Friedman and R. Miranda, Smoothing cusp singularities of small length, Math. Ann. 263 (1983), 185-212.

4. R. Friedman and H. Pinkham, Smoothing of cusp singularities via triangle singularities, Compositio Math. 53 (1984), 303-316.

5. F. Hirzebruch, Hilbert Modular surfaces, Enseign. Math. 19 (1973).

6. M. Inoue, New surfaces with no meromorphic functions II, Complex Analysis and Algebraic Geometry (W. L. Baily and T. Shioda, eds.), Cambridge University Press, London, 1977.

7. H. Laufer, Taut two-dimensional singularities, Math. Ann. 205 (1973).

8. _ _ Deformations of resolutions of two-dimensional singularities, Proc. Rice Conference on Complex Analysis (1972).

9. E. Looijenga, Rational surfaces with an anticanonical cycle, Ann. of Math. 114 (1981).

10. U. Persson, On degenerations of algebraic surfaces, Mem. Amer. Math. Soc. 11 (1977), 189.

11. H. Pinkham, Automorphisms of cusps and Inoue-Hirzebruch surfaces, Compositio Math. 52 (1984).

12. J. Wahl, Smoothing of normal surface singularities, Topology 20 (1981).

Department of Mathematics, Ohio State University at Mansfield, Mansfield, Ohio 44906 